\title{
COMMUTATIVE ALGEBRAS OF HOCHSCHILD DIMENSION ONE
}

\author{
A. R. MAGID
}

Let $R$ be a commutative ring. Call a commutative $R$-algebra $S$ locally separable if every finite set of elements of $S$ is contained in a separable $R$-subalgebra which is finitely generated and projective as an $R$-module. If in addition the subring of $S$ left fixed by the group $\operatorname{Aut}_{R}(S)$ of all $R$-algebra automorphisms of $S$ is precisely $R, S$ is called a locally Galois $R$-algebra. An element $s$ of $S$ is almost separable if for all $g$ in $\operatorname{Aut}_{R}(S) s-g(s)$ is either 0 or not a zero divisor.

THEOREM. Let $T$ be a locally Galois R-algebra with no idempotents except zero and one and let $S$ be a locally separable $R$-subalgebra of $T$ generated by almost separable elements. Then if $S$ is of Hochschild dimension one, $S$ is countably generated as an $R$-module.

This theorem partially answers a question raised by Rosenberg and Zelinsky [5]. A similar result for the case of fields was obtained by MacRae [3]. We break the proof into lemmas, retaining throughout the notation of the hypotheses of the theorem. Unsubscripted tensors are over $R$. Thanks are due to the referee for some valuable suggestions.

Let $G=\operatorname{Aut}_{R}(T) . G$ is the inverse limit of its restrictions to the separable $R$-subalgebras of $T$ [4, Theorem 2, p. 338] and hence has a topology as a profinite group. Let $T$ carry the discrete topology and let $C(G, T)$ be the ring of continuous $T$-valued functions on $G$. There is an $R$-algebra map $f: S \otimes S \rightarrow C(G, T)$ defined by $f(s \otimes t)(g)$ $=s g(t)$.

Lemma 1. $f: S \otimes S \rightarrow C(G, T)$ is a monomorphism.

Proof. Let $x=\sum x_{i} \otimes y_{i}$ lie in the kernel of $f$ and let $V$ be a separable $R$-subalgebra of $S$, finitely generated and projective as an $R$-module, containing each $x_{i}$ and $y_{i}$. Let $W$ be the subalgebra of $T$ generated by the conjugates of $V$ under $G ; W$ is finitely generated and projective as an $R$-module and separable as an $R$-algebra, $\operatorname{Aut}_{R}(W)$ is finite, and the restriction map $G \rightarrow \mathrm{Aut}_{R}(W)$ is onto. (These facts are found in $[4$, Theorem 1, p. 335] and [1, Corollary 3.3, p. 25].) Let $E$ be the ring of all $W$-valued functions on $\operatorname{Aut}_{R}(W)$ and let $h: W \otimes W \rightarrow E$ be defined by $h(w \otimes v)(g)=w g(v)$. By [1, Theorem 1.3,

Received by the editors June 2, 1969. 
p. 18], $h$ is an isomorphism. Moreover, the map of $E$ to $C(G, T)$ induced by the restriction of $G$ to $\operatorname{Aut}_{R}(W)$ and the inclusion of $W$ into $T$ is one-one. $V$, being separable over $R$, is a direct summand of $W$, so the composite $V \otimes V \rightarrow W \otimes W \rightarrow E \rightarrow C(G, T)$ is one-one. The image of $\sum x_{i} \otimes y_{i}$ (in $V \otimes V$ ) under this composite is the same as the image of $x$ under $f$, so that $\sum x_{i} \otimes y_{i}=0$ in $V \otimes V$ and thus also in $S \otimes S$.

For $y$ an element of $S \otimes S$, let $Z(y)$ be the set of all $s$ in $S$ such that $(s \otimes 1-1 \otimes s) y=0$ and let $Z^{\prime}(y)$ be the subring of $S$ left fixed by the set $U$ of all $g$ in $G$ such that $f(y)(g) \neq 0$.

Lemma 2. If $y$ is nonzero, $Z^{\prime}(y)$ is a finitely generated separable $R$-algebra. If $s$ is an almost separable element of $S, s$ is in $Z(y)$ if and only if $s$ is in $Z^{\prime}(y)$.

Proof. Let $H$ be the subgroup of $G$ generated by $U$ and the group $P$ of automorphisms fixing $S$. By [4, Theorem 3, p. 339], $S$ is precisely the subring left fixed by $P$. Thus $Z^{\prime}(y)$ is precisely the subring left fixed by $H$. Since $y$ is not zero, Lemma 1 implies that $U$ is a nonempty open subset of $G$. Thus $H$ is open in $G$ and $[G: H]$ is finite. By [4, Theorem 2, p. 338], then, $Z^{\prime}(y)$ is a separable $R$-algebra, finitely generated as an $R$-module. The second assertion follows directly from Lemma 1.

Proof of Theorem. To say that $S$ has Hochschild dimension one means that the kernel $J$ of the multiplication map of $S \otimes S$ to $S$ is a projective $S \otimes S$-module. Let $\left\{f_{i}, x_{i}\right\}$ be a projective coordinate system for $J$. We may assume that no $f_{i}\left(x_{i}\right)$ is zero. For each $x$ in $J$, only finitely many of the $f_{i}(x)$ are not zero, and since each $f_{i}$ is $S \otimes S$-linear, $f_{i}(x) x_{i}=f_{i}\left(x x_{i}\right)=x f_{i}\left(x_{i}\right)$. For each $i$, let $S_{i}=Z\left(f_{i}\left(x_{i}\right)\right)$ and let $T_{i}=Z^{\prime}\left(f_{i}\left(x_{i}\right)\right)$. Each element $s$ of $S$ lies in all but finitely many of the $S_{i}$, for $s \otimes 1-1 \otimes s$ is in $J$ and by the above only finitely many of the $(s \otimes 1-1 \otimes s) f_{i}\left(x_{i}\right)$ are nonzero. Thus each almost separable element lies in all but finitely many $T_{i}$. There are infinitely many $x_{i}$ : for if not, let $e=\sum f_{i}\left(x_{i}\right)$. For each $s$ in $S$ we have by the properties of a projective coordinate system $s \otimes 1-1 \otimes s=\sum f_{i}(s \otimes 1-1 \otimes s) x_{i}$ $=(s \otimes 1-1 \otimes s) e$. Since $e$ is clearly idempotent and $S$ has no nontrivial idempotents, either $e$ or $1-e$ lies in $J$. In the first case, the map of $S$ to $S \otimes S$ sending $s$ to $(s \otimes 1)(1-e)$ is an $S \otimes S$-inverse to the multiplication map of $S \otimes S$ to $S$ and hence $S$ is of dimension zero, contrary to hypothesis. The second case is similar. Since there are infinitely many $x_{i}$, each $s$ in $S$ lies in some $S_{i}$, and thus each almost separable $s$ lies in some $T_{i}$. Since $S$ is not separable, there are infinitely many $T_{i}$. Now let $s$ be any element of $S$. There are almost separable elements $s_{1}$, $\cdots, s_{n}$ such that $s$ is in $R\left[s_{1}, \cdots, s_{n}\right]$. Each $s_{k}$ is in all but finitely 
many $T_{i}$, hence $s$ is in all but finitely many $T_{i}$. It follows that any countable union of the $T_{i}$ must be all of $S$. Since each $T_{i}$ is finitely generated, $S$ must be countably generated.

If $s$ is an element of $S$ such that $R[s]$ is a separable $R$-subalgebra of $S$ (i.e. $s$ is a separable element) then by [2, Lemma 2.1, p. 467, and Lemma 2.7, p. 469] $s$ is almost separable. Thus the theorem applies when $S$ is generated by separable elements. This will always happen if $R$ is a local ring with infinite residue class field [2, Lemma 3.1, p. 471]. Also, if $T$ is a domain clearly every element of $S$ is almost separable.

Now if $R$ is any connected (i.e. contains no nontrivial idempotents) commutative ring and if $S$ is any connected, locally separable $R$ algebra, $S$ can always be embedded in a connected locally Galois $R$-algebra, namely the separable closure of $R[2, \mathrm{pp} .462-465]$. If $R$ is an integrally closed Noetherian domain, the separable closure of $R$ is also a domain by $[2$, Corollary 4.2, p. 473]. Thus we have

COROLlaRY. Let $R$ be an integrally closed Noetherian domain or a local ring with infinite residue class field. Then any connected, locally separable R-algebra of Hochschild dimension one is countably generated as an $R$-module

\section{REFERENCES}

1. S. Chase, D. Harrison and A. Rosenberg, Galois theory and Galois cohomology of commutative rings, Mem. Amer. Math. Soc. No. 52 (1965), 15-33. MR 33 \#4118.

2. G. Janusz, Separable algebras over commulative rings, Trans. Amer. Math. Soc. 122 (1966), 461-479. MR 35 \#1585.

3. R. MacRae, On cardinality, cohomology, and a conjecture of Rosenberg and Zelinsky, Trans. Amer. Math. Soc. 118 (1965), 243-246.

4. T. Nagahara, A note on Galois theory of commutative rings, Proc. Amer. Math. Soc. 18 (1967), 334-340. MR $34 \# 7580$.

5. A. Rosenberg and D. Zelinsky, Cohomology of infinite algebras, Trans. Amer. Math. Soc. 82 (1956), 85-98. MR 17, 1181.

NorthWEstern UNIVERSITY 